\title{
Critical analysis of antimicrobial and respiratory fixed dose combinations available in Indian market
}

Samidh Shah, Jignesh Patel, Mira Desai, R. K. Dikshit ${ }^{1}$

Department of Pharmacology, B.J. Medical College, ${ }^{1}$ Department of Pharmacology, GCS Medical College, Ahmedabad, Gujarat, India

Address for the Correspondence: Dr. Samidh Shah, Department of Pharmacology, B.J. Medical College, Ahmedabad - 380016 , Gujarat, India.

E-mail: samidhshah@yahoo.com

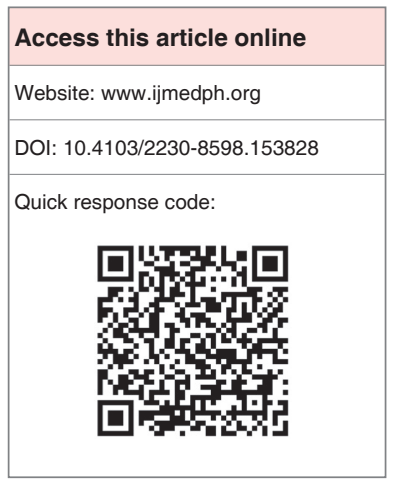

Objective: To analyze the rationality of antimicrobial (AM) and respiratory (RP) fixed dose combinations (FDCs) available in Indian market. Materials and Methods: Antimicrobial and RP FDCs enlisted in Indian Drug Review 2010 and 2013 respectively were analyzed by a pretested validated 8 point criteria tool. Each FDC was assessed for number of active pharmacological ingredients, approval by regulatory authority, listing in World Health Organization (WHO) essential medicine list (EML) or National List of Essential Medicine. While efficacy, safety, pharmacokinetic and pharmacodynamic interactions and advantages of each FDC were analyzed by evidence based literature search. Each criterion was scored one for positive and minus one for negative or unfavorable observation. The total score for the tool was 12 and score $\geq 7$ was considered rational. Results: Of 209 FDCs, 108 were AMs and 101 were RPs. The mean rationality score was $5.41 \pm 1.63$ (95\% Cl, 2.15-8.67). Majority of FDCs were irrational (174) while 35 were rational, and only 12 of these were listed in WHO EML 2013. Out of 108 AM FDCs, 21 (19\%) were rational while $87(81 \%)$ were irrational. Out of 101 RP FDCs, 14 (14\%) were rational while 87 (86\%) were irrational and $24(24 \%)$ with unfavorable pharmacodynamic interactions. Majority of the rational AM FDCs were antiretroviral (6) agents while RP FDCs were indicated for chronic obstructive pulmonary diseases. While majority of irrational FDCs were AM plus steroids, $\beta_{2}$ agonists plus antihistaminics/expectorants/anticholinergics/mast cell stabilizers/leukotriene receptor antagonists. Conclusion: Rationality assessment of AM and RP FDCs reveals that a substantial number of these FDCs in Indian market are irrational. This calls for a close scrutiny of marketed FDCs and educating prescribers to use them with great care and caution. This also indicates a serious review of the regulatory framework for drug manufacturing and marketing.

Key words: Antimicrobial, fixed dose combination, rationality, respiratory

\section{INTRODUCTION}

Fixed dose combination (FDC) is defined as "A combination of two or more active ingredients in a single dosage form in a fixed ratio of doses." FDC products are acceptable when the dosage of each ingredient meets the requirement of a defined population, and the combination has a proven advantage over single compounds administered separately in therapeutic effect, safety and compliance. ${ }^{[1]}$ World Health Organization (WHO) essential medicine list (EML) 2013 includes 24 FDCs and most of them belong to antiretroviral, antitubercular and antimalarial class. ${ }^{[2]}$ Earlier, The Drug and Cosmetic Act (1940) stated "no additional or human data is required and approval to market the product was granted for FDC with an acceptable rationale." This resulted in mushrooming of FDCs in India, accounting for approximately 44\% of domestic pharmaceutical market. ${ }^{[3]}$ However, an Amendment in 2005 clearly stated FDCs as new drugs and must comply the Schedule Y for marketing approval. With this, FDCs have been categorized into two types for regulatory approval. One, any one of the ingredient is new and proposed to be used for the $1^{\text {st }}$ time is considered a new drug. While FDC in which ingredients are individually approved and marketed for certain claims and are proposed to be combined for $1^{\text {st }}$ time in a fixed ratio or if the ratio of the ingredients in an already marketed combination is proposed to be changed for a particular claim is considered as second category. Thus, the marketing approval of FDC requires to submit all necessary 
pharmacological, toxicological and clinical data of individual drug along with the rationale of the combination. Central Drug Standard Control Organization in August 2010 had laid down guidelines for approving the FDCs emphasizing the rationale of combining drugs in a specific ratio. ${ }^{[4]}$ However, there is no available validated tool to assess the rationality. Thus, the present study was aimed to design a tool to assess the rationality of antimicrobial (AM) and respiratory (RP) FDCs available in Indian market.

\section{MATERIALS AND METHODS}

A cross-sectional, observational study was conducted at the Department of Pharmacology, B. J. Medical College, Ahmedabad, Gujarat. The data

1. Active pharmacological ingredient along with strength ......................

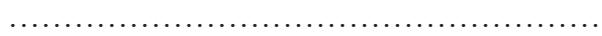

2. API

\begin{tabular}{|c|c|c|c|}
\hline & API & & \\
\hline 1 & Approved by DCGI & Yes $(+1)$ & No $(-1)$ \\
\hline 2 & $\begin{array}{l}\text { Ingredient: Banned or } \\
\text { controversial }\end{array}$ & Yes $(-1)$ & No $(+1)$ \\
\hline
\end{tabular}

3. Listing in EML

WHO/National/Both/None

$(+1) \quad(0)$

4. Efficacy (text book/reference book/pub med/medline/ other)

\begin{tabular}{cccc}
1 & API & Yes $(+1)$ & No $(0)$ \\
2 & FDC & Yes $(+1)$ & No $(0)$ \\
\hline API $=$ Active & pharmacological ingredient, & FDC & Fixed dose combination
\end{tabular}

5. Safety (text book/reference book/pub med/medline/other) 1 API Yes (+1) No (0)

2 FDC $\quad$ Yes $(+1) \quad$ No $(0)$

$\mathrm{API}=$ Active pharmacological ingredient, $\mathrm{FDC}=$ Fixed dose combination

6. Pharmacokinetic (absorption/distribution/metabolism/ excretion/BA/BE/ $1 / 2$ )

- Interaction Favorable/Unfavorable/Not affected

$(+1) \quad(-1)$

7. Pharmacodynamic-M/A of each ingredient Similar (0)/Different $(+1)$

8. Advantage of FDC
- Reduced
Yes $(+1) / \mathrm{No}(0)$
- Less ADR
Yes $(+1) /$ No $(0)$
- Convenient (frequency or pill count) Yes $(+1) / \mathrm{No}$ (0)

Total score: 12

Score $\geq 7$ : Rational FDC

Score $\leq 6$ : Irrational FDC

Figure 1: Tool to assess the rationality of fixed dose combinations available in Indian market were collected from an annual Drug Compendium entitled "Indian Drug Review” (IDR) 2010 and 2013 that enlist most of the medicines commercially available in India during a particular year.

Antimicrobial and RP FDCs enlisted in IDR 2010, and 2013 respectively were assessed for rationality. A tool was designed using WHO guidelines for registration of fixed dose combination medicinal products. ${ }^{[1]}$ The tool consisted of eight point criteria [Figure 1]. The criteria in the tool included active pharmacological ingredient (API) with strength, its efficacy, safety, approval by regulatory authority, listing in WHO EML or National List of Essential Medicines of (NLEM). The evidence for efficacy and safety of the individual API and their combination was searched using standard textbooks, reference books of pharmacology and medicine. In addition, authentic web sources like PubMed data base, Google scholar and Cochrane data base were also used. Pharmacokinetic and pharmacodynamic interactions were checked using Medscape drug interaction checker. Advantages of FDC over individual API in the form of dose reduction, less adverse drug reactions and reduced frequency of administration were assessed by above mentioned evidence based literature search.

As per subject experts opinion form industry and academia each criterion was scored plus one $(+1)$ for positive and minus one $(-1)$ for negative or unfavorable observation. The total score ranged from 1 to 12 and score $\geq 7$ was considered rational. Further the tool was validated by assessment of FDCs already approved by WHO EML 2013 whereby the minimum score obtained of these was seven. The data was entered in Microsoft excel sheet and analyzed using appropriate statistical tests.

\section{RESULTS}

Out of 209 FDCs, 108 belonged to AM group and 101 to RP group. The mean rationality score of AM FDCs was $4.91 \pm 1.72$ while that of RP FDCs was $5.92 \pm 1.55$. Majority of AM FDCs contained two APIs (90) while majority of RP FDCs contained 3 or $>3$ active ingredients [Table 1 and Figure 2]. The rationality score for 12 AM FDCs listed in WHO EML was $\geq 7$ [Table 2]. In

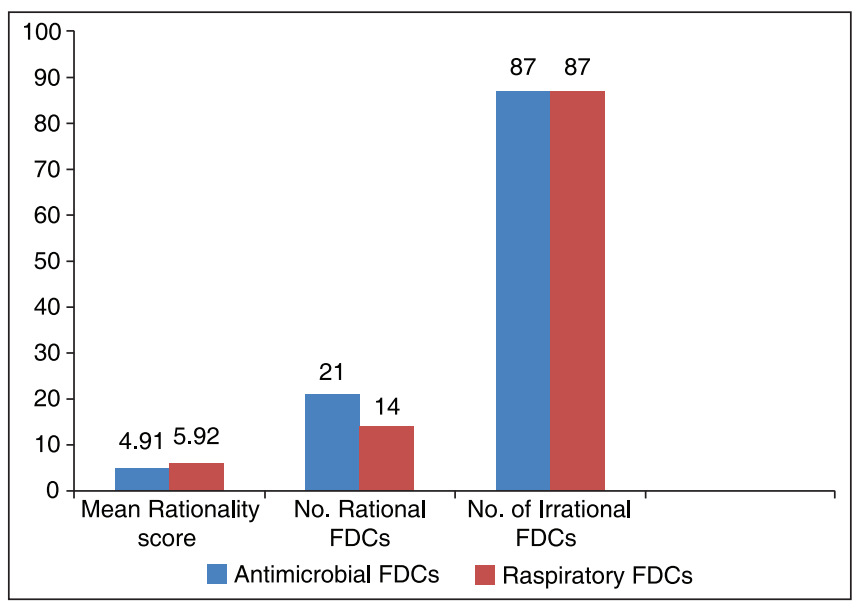

Figure 2: Rationality score and rational and irrational fixed dose combinations in both categories 
addition, there were 9 other AM FDCs and 14 RP FDCs scored $\geq 7$ as per our tool [Table 3].

Of 108 AM FDCs, 21 (19\%) were rational and 87 (81\%) were irrational. Out of 21 rational FDCs, 12 were listed in WHO EML 2013 while out of 87 irrational AM FDCs, none was listed in WHO

\begin{tabular}{lcc}
$\begin{array}{l}\text { Table 1: Assessment of antimicrobial and } \\
\text { respiratory FDCs using rationality tool }\end{array}$ \\
\hline $\begin{array}{l}\text { Antimicrobial } \\
\text { FDCs }(\boldsymbol{n}=\mathbf{1 1 1}) \\
(\%)\end{array}$ & $\begin{array}{c}\text { Respiratory } \\
\text { FDCs }(\boldsymbol{n}=\mathbf{1 0 1}) \\
(\%)\end{array}$ \\
\hline Mean rationality score & $4.91 \pm 1.72$ & $5.92 \pm 1.55$ \\
Minimum score & 2 & 1 \\
Maximum score & 12 & 10 \\
Number of rational FDCs & $21(19)$ & $14(14)$ \\
Number of irrational FDCs & $87(81)$ & $87(86)$ \\
Number of API in each FDC & & \\
2 & 90 & 36 \\
3 & 18 & 28 \\
$\geq 4$ & 0 & 37 \\
FDCs enlisted in WHO EML & 12 & $\mathrm{Nil}$ \\
\hline
\end{tabular}

$\mathrm{FDC}=$ Fixed dose combination, $\mathrm{EML}=$ Essential medicine list, $\mathrm{WHO}=$ World health organization, $\mathrm{API}=$ Active pharmacological ingredients

\begin{tabular}{ll} 
Table 2: List of FDCs scored $\geq 7$ (rational) as per \\
tool and enlisted in WHO EML $2013(n=12)$ \\
\hline \multicolumn{1}{c}{ Antimicrobial FDCs $(n=12)$} \\
\hline $\begin{array}{ll}\text { Imipenem+cilastin } & \text { Artesunate+amodiaquine } \\
\text { Lamivudine+nevirapine+stavudine } & \text { Artemether+lumifantrine } \\
\text { Lopinavir+ritonavir } & \text { Lamivudine+zidovudine } \\
\text { Amoxicillin+clavulanic acid } & \text { Lamivudine+zidovudine+nevir } \\
& \text { apine } \\
\text { Tenofovir+emtricitabine } & \text { Rifampicin+isoniazid+ethambutol } \\
\text { Tenofovir+emtricitabine+efavirenz } & \text { Sulfamethoxazole+trimethoprim } \\
\text { Respiratory FDCs }(n=0)\end{array}$
\end{tabular}

None

$\mathrm{FDC}=$ Fixed dose combination, $\mathrm{EML}=$ Essential medicine list, $\mathrm{WHO}=$ World health organization

\begin{tabular}{|c|c|}
\hline \multicolumn{2}{|c|}{ Antimicrobial FDCs $(n=9)$} \\
\hline Stavudine+lamivudine & Loteprednol+tobramycin \\
\hline Atazanavir+ritonavir & $\begin{array}{l}\text { Hyluronic acid+silver } \\
\text { sulfadiazine }\end{array}$ \\
\hline Tenofovir+lamivudine+efavirenz & Mometasone+fusidic acid \\
\hline Clindamycin+isotretinoin & Ketoconazole+zinc \\
\hline \multicolumn{2}{|l|}{ Albendazole+ivermectin } \\
\hline \multicolumn{2}{|c|}{ Respiratory FDCs ( $n=14)$} \\
\hline Budesonide+formoterol & Salmeterol+beclometasone \\
\hline Doxophylline+terbutaline & Salbutamol+theophylline \\
\hline Formoterol+fluticasone & Salmeterol+fluticasone \\
\hline Formoterol+tiotropium & Terbutaline+theophylline \\
\hline Ipratropium+salbutamol & Formoterol+tiotropium \\
\hline Montelukast+levocetirizine & Fexofenadine+pseudoephedrine \\
\hline Beclometasone+salbutamol & Pseudoephedrine+desloratadine \\
\hline
\end{tabular}

EML 2013. Majority of the rational AM FDCs were antiretroviral (6) agents. Favorable pharmacokinetic interactions were observed in 4 rational FDCs while only two FDCs had advantages of less adverse drug reactions (ADRs) when combined as compared to individual ingredient. Majority of irrational AM FDCs (87, 81\%) were combination of AM plus steroids (21) followed by cephalosporin plus betalactamase inhibitor (20), antibacterial plus antiamoebic plus antifungal agents (10) and combinations of ingredients of same class and antibacterial with lactobacilli or serratiopeptidase. Surprisingly, two FDCs contained controversial ingredients such as piperine and coal tar.

Out of 101 RP FDCs, 14 (14\%) were rational and 87 (86\%) were irrational. However, none of rational RP FDCs scoring $\geq 7$ was listed in WHO EML 2013. The rational FDCs were combinations of $\beta_{2}$ agonists plus corticosteroids/anticholinergics/methylxanthines. Favorable pharmacodynamic interactions were observed in 3 rational FDCs. Moreover, three FDCs also had advantage of having less ADRs in combination than single ingredient. The irrational FDCs were combinations of $\beta_{2}$ agonists plus antihistaminics plus expectorants/anticholinergics/sympathomimetics.

\section{DISCUSSION}

The results of this study show that a large number of AM and RP FDCs are available in Indian market. Unfortunately, their rationality assessment showed that majority had a score $<7$ indicating towards irrationality.

The rational AM FDCs were antiretroviral, antitubercular and antimalarial class of drugs with proven efficacy, safety, pharmacokinetic and pharmacodynamic advantages. ${ }^{[5,6]}$ While $81 \%$ of AM FDCs were irrational, with no justification of combining the ingredients. Our results are in conformity with the observation made by Rayasam et al. in 2013 to study prescribing pattern of FDCs among practitioners in central India. ${ }^{[7]}$ The most pressing concern with irrational FDCs was illogical combination of antibacterial with corticosteroid which decreases the immunity and increases the susceptibility to infections. Moreover, combination of cephalosporin with beta lactamase inhibitor is also not justified as beta lactamse inhibitors prevent destruction of beta lactam ring in penicillin group of antibiotics and thus widens the antibacterial spectrum of latter. However, these are not effective against the extended spectrum cephalosporins such as ceftazidime, ceftriaxone, cefotaxime. ${ }^{[8]}$ A large number of AM FDCs were fashionable combination of antibacterial with antiamoebic and/or antifungal agents. Amoebic and bacterial infection rarely coexist and such combination not only encourage resistance, but also expose patients to unnecessary risk of adverse drug reactions. ${ }^{[9]}$ Such FDCs have not been recommended in any standard books, but continue to be available in Indian market. Availability of such products reduces the attempt for a precise diagnosis and only adds to the cost of therapy. It is a wellknown fact that lactobacilli as a single agent is used for diarrhea. However, none of the combination of antibacterial plus lactobacilli 
had adequate dose (5-10 billion) required to decrease antibacterial induced diarrhea. In addition, many FDCs were combined with serratiopeptidase, an enzyme claimed to promote rapid resolution of inflammation. To our surprise, we could not find any evidence in published literature such as standard books or peer reviewed scientific journals supporting this claim.

On the other hand, no RP FDC has been listed in WHO EML. Majority of RP FDCs $(86 \%)$ in our study were irrational, many of them targeting cough and the common cold, a self-limiting condition that does not require medicine to cure or prevent it. ${ }^{[10]}$ Similar observations have been reported by Roy et al..$^{[11]}$ and Desai et al..$^{[10]}$

Interestingly, our tool found 14 RP FDCs rational with respect to efficacy, safety, and compliance. For example, the combination of long acting $\beta_{2}$ agonist with corticosteroid is rational because corticosteroids increase the expression of $\beta_{2}$ receptors by increasing gene transcription and reduces the adverse effects of $\beta 2$ agonist, whereas $\beta_{2}$-agonists potentiates the local anti-inflammatory actions of corticosteroids by increasing nuclear localization of glucocorticoid receptors and additive suppression of inflammatory mediator release. ${ }^{[12]}$ Further, combination of methylxanthines plus $\beta_{2}$-agonists potentiates the therapeutic effect with fewer side effects as compared to $\beta_{2}$-agonists alone. ${ }^{[13,14]}$ Literature states that combination therapy with $\beta_{2}$-agonists and anticholinergics is also effective with improvement of dynamic lung function and good safety profile in patients of chronic obstructive pulmonary diseases. ${ }^{[15]}$ FDCs of montelukast with antihistaminics were also found to be rational due to its enhanced and complementary pharmacological and clinical effects leading to a reduction in the day and night time symptoms effectively in patients of allergic rhinitis. ${ }^{[16]}$

A great discrepancy was observed in the total number of AM and RP FDCs available in Indian market as compared to WHO EML and NLEM. The WHO EML 2013 includes a total of $18 \mathrm{AM}$ FDCs out of total 120 AMs with nil from RP system. Thus, it is almost 11 times the numbers of FDCs to treat infectious and RP diseases in India.

Although there has been no defined rule for the permissible maximum number of constituents in the FDCs, any FDC containing $>3$ ingredients should be looked at watchfully. Majority of AM FDCs had two ingredients, albeit, majority of RM FDCs had 3 or $>3$ ingredients. US FDA states that there is no rationale of combining $>3$ ingredients. ${ }^{[1]}$

A commercial and commonly used annual drug compendium among the prescribers was used for data collection and source of information for formulations available in Indian market. A possibility of lack of complete information cannot be ruled out. Nevertheless, the compendium still reflects the actual scenario of marketed drug product of the country.

Thus, it can be concluded that a large number of AM and RP irrational FDCs to treat clinical conditions are available in Indian market. It is indeed very unfortunate and unethical to expose the innocent patients to medicines with unproven efficacy and safety. This calls for a close scrutiny of marketed FDCs and educating prescribers to use them with great care and caution. This also indicates a serious review of the regulatory framework for drug manufacturing and marketing.

\section{REFERENCES}

1. Guidelines for registration of fixed-dose combination medicinal products Geneva. World Health Organization 2005. Report number 929.

2. Model List Essential Medicine $18^{\text {th }}$ list. Geneva. World Health Organization; 2013, p. 45.

3. Ghose Jayati. "Combo drug crackdown set to bleed Pharma Inc". The Indian EXPRESS: New Delhi; Sat, Sep 14, 2013.

4. Guidelines for Industry on Fixed dose combinations draft guidelines: New Delhi. Ministry of Health and Family Welfare. Government of India 2010 p.38.

5. Pujari S, Patel A, Patel K, Dravid A, Patel J, Mane A, et al. Safety and long-term effectiveness of generic fixed-dose formulations of nevirapinebased HAART amongst antiretroviral-naïve HIV-infected patients in India. Geneva World Health Organization; 2003, p. 99-112.

6. Flexner $\mathrm{C}$. The pharmacological basis of therapeutics. Chapter, antiretroviral agents and treatment of HIV infection. $11^{\text {th }}$ ed. New York: Macgraw-Hill; 2006. p. 1290-1.

7. Rayasam SP, Dudhgaonkar SS, Dakhale GN, Hire RC, Deshmukh PS, Gaikwad NN. The irrational fixed dose combinations in the Indian drug market: An evaluation of prescribing pattern using WHO guidelines. Int $\mathrm{J}$ Basic Clin Pharmacol 2013;2:52-457.

8. Lee NL, Yuen KY, Kumana CR Beta-lactam antibiotic and beta-lactamase inhibitor combinations. JAMA 2001;285:386-8.

9. Sreedhar D, Janodia MD, Ligade VS, Mohapatra S, Ganguly R, Udupa N. Fixed dose combinations: Rational or irrational? Curr Sci 2008;95:581-3.

10. Desai P, Patel S, Shah R, Desai S. A comprehensive evaluation of rationality of cough and cold medicines available in Indian market. J Indian Med Assoc 2013;111:94-8.

11. Roy V, Malhotra R, Tayal V, Bansal A, Gupta KS. Fixed-dose combinations for cough and common cold in India: An assessment of availability and rationality. Fundam Clin Pharmacol 2011;25:258-66.

12. Barnes PJ. Scientific rationale for inhaled combination therapy with long-acting beta2-agonists and corticosteroids. Eur Respir J 2002;19:182-91.

13. Waldeck B. Beta-adrenoceptor agonists and asthma-100 years of development. Eur J Pharmacol 2002;445:1-12.

14. Rosenburg J. Clinical-pharmacokinetic aspects of prolonged effect duration as illustrated by beta2-agonists. Eur $\mathrm{J}$ Clin Pharmacol 2002;58:S1-21.

15. Di Marco F, Verga M, Santus P, Morelli N, Cazzola M, Centanni S. Effect of formoterol, tiotropium, and their combination in patients with acute exacerbation of chronic obstructive pulmonary disease: A pilot study. Respir Med 2006;100:1925-32.

16. Gupta V, Matreja PS. Efficacy of Montelukast and Levocetirizine as Treatment for Allergic Rhinitis. J Aller Ther 2010. 1:103. doi:10.4172/21556121.1000103 [Last accessed on 2014 Sep 25].

17. Hussein SH. Cough mixtures. [Online]. 1996. Available from:URL:http:// www.prn2.usm.my/mainsite/bulletin/sun/1996/sun39.html [Last accessed on 2014 Sep 25].

How to cite this article: Shah S, Patel J, Desai M, Dikshit RK Critical analysis of antimicrobial and respiratory fixed dose combinations available in Indian market. Int $\mathrm{J}$ Med Public Health 2015;5:161-4.

Source of Support: Nil, Conflict of Interest: None declared. 